\title{
Green open location-routing problem considering economic and environmental costs
}

\author{
Eliana M. Toro ${ }^{a}$, John F. Franco ${ }^{b}$, Mauricio Granada Echeverri ${ }^{\mathrm{c}^{*}}$, Frederico G. Guimarães ${ }^{\mathrm{d}}$ and Ramón \\ A. Gallego Rendón ${ }^{\mathrm{e}}$
}

${ }^{a}$ Facultad de Ingeniería Industrial, Universidad Tecnológica de Pereira. Pereira, Colombia

${ }^{b}$ Universidade Estadual Paulista Júlio de Mesquita Filho, UNESP, Ilha Solteira, Brazil

${ }^{c}$ Programa de Ingeniería Eléctrica, Facultad de Ingenierías, Universidad Tecnológica de Pereira., Pereira, Colombia

${ }^{d}$ Department of Electrical Engineering, Universidade Federal de Minas Gerais, UFMG, Belo Horizonte, Brazil

ePrograma de Ingeniería Eléctrica, Facultad de Ingenierías, Universidad Tecnológica de Pereira., Pereira, Colombia

\section{CHRON I C L E}

\section{Article history:}

Received April 262016

Received in Revised Format

August 162016

Accepted October 312016

Available online

October 312016

Keywords:

Open Location-Routing Problem

Green Vehicle Routing Problem

Green logistics

Mixed-Integer Linear

Programming

Vehicle Routing Problem

\begin{abstract}
A B S T R A C T
This paper introduces a new bi-objective vehicle routing problem that integrates the Open Location Routing Problem (OLRP), recently presented in the literature, coupled with the growing need for fuel consumption minimization, named Green OLRP (G-OLRP). Open routing problems (ORP) are known to be NP-hard problems, in which vehicles start from the set of existing depots and are not required to return to the starting depot after completing their service. The OLRP is a strategic-level problem involving the selection of one or many depots from a set of candidate locations and the planning of delivery radial routes from the selected depots to a set of customers. The concept of radial paths allows us to use a set of constraints focused on maintaining the radiality condition of the paths, which significantly simplifies the set of constraints associated with the connectivity and capacity requirements and provides a suitable alternative when compared with the elimination problem of sub-tours traditionally addressed in the literature. The emphasis in the paper will be placed on modeling rather than solution methods. The model proposed is formulated as a bi-objective problem, considering the minimization of operational costs and the minimization of environmental effects, and it is solved by using the epsilon constraint technique. The results illustrate that the proposed model is able to generate a set of trade-off solutions leading to interesting conclusions about the relationship between operational costs and environmental impact.
\end{abstract}

\section{Introduction}

The open vehicle routing problem (OVRP) was first proposed in the early 1980s when there were cases where a delivery company did not own a vehicle fleet or its private fleet was inadequate for fully satisfying customer demand (Schrage, 1981; Bodin et al., 1983). Therefore, contractors who were not employees of the delivery company used their own vehicles for deliveries. In these cases, vehicles were not required to return to the central depot after their deliveries because the company was only concerned

* Corresponding author

E-mail: magra@utp.edu.co (M. G. Echeverri)

(C) 2017 Growing Science Ltd. All rights reserved. doi: $10.5267 / j$. ijiec.2016.10.001 
with reaching the last customer. Compensation was not given for any driving outside of meeting this goal. Thus, the goal of the OVRP is to design a set of Hamiltonian paths to satisfy customer demand. During the last decade, consumers, businesses and governments have increased their attentions to the environment. Society in general is becoming increasingly aware and concerned of the environmental impacts of human activities and the indiscriminate use of natural resources. A growing interest is being perceived in companies to assess and reduce the environmental impacts of their products and services (Daniel et al., 1997; Frota Neto et al., 2009). In this context, the transportation industry has a significant effect on the planet, because of the large quantity of fuel used in its regular operations and the environmental consequences and greenhouse effects of fuel consumption and pollution. As a consequence, Green Logistics and Green Transportation have emerged in all levels of supply chain management, with growing value to researchers and organizations, motivated by the fact that current logistics centered on economic costs without accounting for the negative impacts on the environment is not sustainable in the long term (Lin et al., 2014).

During the last few years, many logistics and operations research problems have been extended to include environmental issues and costs related to the environmental impacts of industrial and transportation activities (Bektas \&d Laporte, 2011; Erdogan \& Miller-Hooks, 2012; Demir et al., 2014; Lin et al., 2014). In this paper, we present a bi-objective mathematical model that integrates Open Location Routing Problem (OLRP) and the minimization of fuel consumption, named Green OLRP (G-OLRP). Considering that the vehicles do not return to the depots, we assert that the solution to the OLRP should be formed by radial paths, which allows us to propose a set of new constraints focused on maintaining the radiality condition of the paths, hence simplifying the set of constraints associated with the connectivity and capacity requirements.

Briefly, the OLRP can be stated as the following graph theoretic problem. Let $G=(V, A)$ be a complete and directed graph, where $V=I \cup J$ is the vertex set and $A$ is the $\operatorname{arc}$ set. Vertex set $I=\{1,2, \ldots, m\}$ represents the set of candidate capacitated depots to be installed. Vertex set $J=\{m+1, m+2, \ldots, m+$ $n$ \} represents the customers to be served. Each customer $j \in J$ is associated with a known non-negative demand of goods $D_{j}$ to be delivered. Each candidate depot $i \in I$ has a fictitious demand $D_{i}=0$ and has an unlimited fleet of identical vehicles with the same positive capacity, denoted as $Q$. Note that for feasibility, the vehicle must meet the criterion that $0<D_{j} \leq Q$ for all customers $j$. In addition, each candidate depot $i$ has limited storage capacity of goods and a set-up cost, respectively denoted as $W_{i}$ and $O_{i}$. A nonnegative traveling cost $C_{i j}$ and non-negative traveling distance $T_{i j}$ are associated with each $\operatorname{arc}(i, j) \in A$, and a non-negative fixed cost $F$ is associated with the use of each vehicle. Thus, the OLRP consists of finding a collection of routes of minimum travelling cost connected to a set of depots with minimum setup cost to satisfy the following:

- Each customer vertex is visited by exactly one route.

- Each route begins at a depot and ends at a client, thus forming radial paths only.

- Each customer has to be fully served when visited (no fractional deliveries).

- The sum of the demands of the vertices visited by a route cannot exceed the vehicle capacity.

In the G-OLRP, in addition to the operational costs, a second objective function is included, which considers emissions generated due to fuel consumption in the routes performed. The mathematical model for the computation of fuel consumption and total emissions is based on the forces acting on each vehicle during its operation. Given the inherent difficulty of aggregating these objectives into a global criterion, we formulate the problem as a bi-objective one. The Pareto optimal solutions for the problem can be found and then analyzed a posteriori by decision-makers. This model corresponds to a mixed integer linear formulation and was implemented in AMPL (Fourer et al., 2002) and solved with CPLEX (called with the optimality gap option equal to $0 \%$ ), using a work station with an Intel i7 870 processor.

The main contributions of this paper are: 
- The OLRP is extended in the proposed G-OLRP, considering the environmental impact in terms of fuel consumption minimization.

- Because it is an approach that focuses on radiality of the paths, the proposed model is well suited to ORP, and the results are of high quality and performance.

- This paper presents a contribution to the discussion of OLRP, which is a new problem insufficiently addressed in the literature.

- This paper presents a contribution to the discussion of green VRP, by considering the integrated location of multiple deposits and open routing of multiple vehicles.

- The radial characteristic of the solution-paths may be extended to other types of problems, such as the VRP with backhauls (VRPB) and the electric vehicle routing problem (EVRP), which are all in the context of Green Transportation.

\section{Literature Review}

In practice, the OVRP formulation represents situations, such as: home delivery of packages and newspapers, school bus routing, routing of coal mines material or shipment of hazardous materials (Braekers et al., 2015). When considering that the vehicles depart from a set of available depots and finish their deliveries at their last customers, the OVRP is generalized to the case known as Multi-Depot OVRP (MDOVRP). Tarantilis and Kiranoudis (2002) proposed the MDOVRP for the first time within the context of food distribution. Since this problem is a generalization of the OVRP, the MDOVRP belongs to the NP-hard class; therefore, heuristic methods are commonly presented in the literature to find good quality solutions quickly (Dueck \& Scheuer, 1990; Ho et al., 2008; Mirabi et al., 2010; Liu \& Jiang, 2012; Yao et al., 2014).

Liu and Jiang (2012) proposed a hybrid genetic algorithm for the MDOVRP; however, the authors additionally proposed a mixed integer programming (MIP) formulation, which is the first formal mathematical definition of the problem. The literature on exact approaches for the MDOVRP is sparse and has received very little attention from researchers. The most recent MIP formulation was reported by Lalla-Ruiz et al. (2016), in which a new mixed integer programming formulation was presented and compared with the unique MIP formulation given in the literature at that time (Liu \& Jiang, 2012). The authors focused on the improvements and extensions of the Miller-Tucker-Zemlin subtour elimination constraints, always considering that each vehicle departs from one of the available depots and finishes at the last customer it serves. As already mentioned above, when in the OVRP a set of candidate depots to be installed is considered, the resulting problem is known as the OLRP. This problem was recently introduced by $\mathrm{Yu}$ and Lin (2015), who also proposed an exact mathematical model to solve scenarios up to 50 clients and five depots with gaps close to zero. Additionally, they proposed a heuristic algorithm based on simulated annealing, which consists of two stages. First, an initial solution was constructed with a greedy approach, and second the initial solution was improved with a simulated annealing procedure with three search mechanisms: exchange, insertion and 2-opt movements. The proposal was also validated with adapted cases from Barreto et al. (2007), Prins et al. (2006) and Tuzun and Burke (1999).

About green issues in vehicle routing, the reduction of indirect green-house gases emissions, addressed in the vehicle routing problem, represents one of the most common objectives to be optimized. The cost of a route depends on several factors that can be divided into two sets. In the first set are included: distance, weight, speed, path conditions, a percentage of fuel that is generally associated to the unit of distance, and fuel costs. The second set of factors does not have direct relationship on the travel programming and includes tire and vehicle depreciation, maintenance, driver wages, taxes, among others (Palmer, 2007; Boriboonsomsin et al., 2010). Comparing the two sets, the first set of factors are directly related to fuel consumption and therefore can be considered as a variable cost or cost of fuel. Also, if other factors remain constant, fuel consumption depends mainly on the distance and load. Entities for environmental impact analysis in the transport sector indicate that there is a strong correlation between the gross vehicle weight and distance traveled using given amount of fuel, see for instance Xiao et al. 
(2012). Kara et al. (2007) is one of the first publications that considers minimizing the fuel consumption where they define the Energy Minimizing Vehicle Routing Problem (EMVRP) as the CVRP in which the objective function is a product of the total load (including the weight of the empty vehicle) and the length of the arc. Figliozzi (2010) compares different levels of traffic congestion and vehicle speeds, to formulate and solve the problem called Emissions Vehicle Routing Problem (EVRP). The problem is an extension of the VRPTW. Greedy heuristic is the solution technique used by the author.

In Bektas and Laporte (2011), the authors consider factors such as speed, vehicle load and travel costs. The load and travel speed are factors that can be controlled. They have submitted four mathematical formulations for the Pollution Routing Problem (PRP) by considering time windows, speed, load, velocity. Branch-and-Cut is the solution technique chosen, using CPLEX 12.1. Suzuki (2011) develops an approach to the time-constrained, multiple stop, truck routing problem that minimizes the fuel consumption and pollutants emission to solve the traveling salesman problem with time windows (TSPTW). Their results suggest the approach may produce up to $6.9 \%$ in fuel savings over existing methods. The solution technique used was the compressed annealing. Xiao et al. (2012) define the Fuel Consumption Vehicle Routing Problem (FCVRP) and propose Fuel Consumption Rate (FCR) as a load dependent function, adding it to the classical CVRP to extend traditional studies on CVRP with the objective of minimizing fuel consumption. The methodology for solving the problem was based on the simulated annealing algorithm with a hybrid exchange rule to solve it. Their results show that the FCVRP model can reduce fuel consumption by $5 \%$ on average compared to the CVRP model.

Erdogan and Miller-Hooks (2012) introduce the Green Vehicle Routing Problem (G-VRP). The G-VRP is formulated as a mixed integer linear program. The solution method is based on two construction heuristics and the Modified Clarke and Wright Savings formulation of Bektas and Laporte (2011). Two construction heuristics, the Modified Clarke and Wright Savings heuristic and the Density-Based Clustering Algorithm, and a customized improvement technique, are developed. Results of numerical experiments show good performance of the heuristics. Moreover, problem feasibility depends on customer and station location configurations. Pradenas et al. (2013) formulate a model with emissions of greenhouse gases for the VRPB problem (VRP with Backhauls). Ubeda et al. (2011) present a case study considering environmental criteria based on real estimations. Other approximations that use metaheuristics can be found in Demir et al. (2012) and Jemai et al. (2012). Demir et al. (2014) propose the bi-objective Pollution Routing Problem (PRP), as an extension of the PRP, which consists of routing a number of vehicles to serve a set of customers, and determining their speed on each route segment. Two objective functions related to minimization of fuel consumption and driving time are proposed. Several multi-objective optimization techniques are developed and tested for the problem, finding tradeoffs between fuel consumption and driver times.

Kücükoglu et al. (2013) present the G-CVRP optimization model, in which fuel consumption is computed considering the vehicle technical specifications, vehicle load and the distance. Fuel consumption equation is integrated to the model through a regression equation proportional to the distance and vehicle load. The GCVRP optimization model is validated by various instances with different number of customers. The authors present a mixed integer programming model, solving it with Gurobi 5.10. Recently, Lin et al. (2014) and Toro et al. (2016) presented an extensive literature review on Green Vehicle Routing Problems.

\section{Proposed Model for the Green OLRP}

In this section we introduce the proposed bi-objective formulation for the G-OLRP. In the first subsection, the mathematical model used to compute the fuel consumption of a vehicle between two nodes is presented. The model is developed based on the forces acting on the vehicle and a detailed extension of this model can be found in Golden et al. (2008). In the second subsection, the model for the G-OLRP is presented. 


\subsection{Computation of fuel consumption and total emission}

In Toth and Vigo (2014), the analytical models for fuel consumption are classified into three classes, namely (i) emission factor models, (ii) average speed models, and (iii) modal models. In this paper an emission factor model is used which is expressed per unit of distance assuming constant speed. Eq. (1) represents one of the objectives of G-OLRP, consisting in the minimization of the fuel consumption and the total emission associated with this fuel consumption.

$$
\Psi_{2}=\alpha E \sum_{i, j \in V} T_{i j} s_{i j}+\gamma E \sum_{i, j \in V} T_{i j} l_{i j} .
$$

The expression for the fuel consumption is formed by two parts. The first part, multiplied by $\alpha$, corresponds to the amount of energy required considering the unloaded vehicle, where $s_{i j}$ are the active arcs. The second term, multiplied by $\gamma$, corresponds to the amount of extra energy required considering the load carried on that arc, where $l_{i j}$ represents the flow of goods transported by a vehicle through the $\operatorname{arc}(i, j) \in A . \alpha$ is a parameter representing how much energy an unloaded vehicle spends in crossing the arc and is given in $\mathrm{J} / \mathrm{km} . \gamma$ is a parameter representing the additional energy (per unit of load) that a loaded vehicle spends in that arc and is given in $\mathrm{J} / \mathrm{km}$-ton. Finally, both terms are multiplied by $E$, which is the total emission per unit of energy $(\mathrm{kg}$ of $\mathrm{CO} 2 / \mathrm{J})$, giving the total emission associated with the solution, which is calculated as:

$$
E=E 1 \times E 2,
$$

where $E_{1}$ is a conversion factor representing the amount of fuel required (gallons/J) and $E_{2}$ is another conversion factor representing the amount of emission per unit of fuel ( $\mathrm{kg}$ of $\mathrm{CO} 2 / \mathrm{gallons})$. The developed model for computing emission is linear and defined as an objective in our formulation.

\subsection{Problem formulation}

The model is a two-index vehicle flow formulation that uses two binary decision variables:

$-s_{i j}=1$ if $\operatorname{arc}(i, j) \in A$ is used and takes value 0 otherwise

$-y_{i}=1$ if a depot is set-up at vertex $i \in I$

Additionally, a real variable $l_{i j}$ that represents the flow of goods transported by a vehicle through the arc $(i, j) \in A$ is used. The standard CLRP is often criticized because its objective function combines facility locations determined at a strategic level, while vehicle routes are optimized at the operational level. For this reason, we propose to put all the values in the same time horizon according to that opening cost and route cost by considering the Net Present Value (NPV) to the lessee, as expressed in (3), see (Trigeorgis, 1996):

$$
N P V=V_{0}-\sum_{t=0}^{N} \frac{I_{t}}{(1+r)^{t}} \equiv V_{0}-I
$$

with

$$
\begin{aligned}
& I_{t}=L_{t}(1-T)+D_{t} T \\
& r=r_{B}(1-T)
\end{aligned}
$$

In these equations, $V_{0}$ is the current value (cost) of the leased asset, $L_{t}$ is the lease rental payment at time $t, D_{t}$ is the depreciation expense at time $t, T$ is the lessee's effective corporate tax rate, $r_{B}$ is the before tax cost of borrowing, and $N$ is the life, i.e. maturity of the lease. In this way, $O_{i}^{N P V}$ represents the NPV of the leasing cost associated to the use of a facility $i \in I$ and $F^{N P V}$ is the NPV of the leasing cost associated to the use of a vehicle, assuming $F_{k}=F, \forall k \in K$. The two-index vehicle flow formulation for the biobjective OLRP is defined as follows: 


$$
\begin{aligned}
& \min \Psi_{1}=\sum_{i \in V} C_{i j} \cdot s_{i j}+\sum_{i \in I} O_{i}^{N P V} \cdot y_{i}+\sum_{\substack{i \in I \\
j \in V}} F^{N P V} \cdot s_{i j} \\
& \min \Psi_{2}=\alpha E \sum_{i, j \in V} T_{i j} s_{i j}+\gamma E \sum_{i, j \in V} T_{i j} l_{i j}
\end{aligned}
$$

subject to :

$$
\begin{aligned}
& \sum_{i \in V} s_{i j}=|J| \\
& \sum_{i \in V}^{j \in V} l_{i j}=\sum_{k \in V} l_{j k}+D_{j} \quad \forall j \in J \\
& \sum_{i \in V}^{i \neq j} s_{i j}=1 \quad \forall j \in J \\
& \sum_{k \in J} s_{j k} \leq \sum_{i \in V} s_{i j} \quad \forall j \in J \\
& s_{i j}+s_{j i} \leq 1 \quad \forall i, j \in V \\
& \sum_{i \in I} y_{i} \geq \sum_{j \in J} D_{j} / \sum_{i \in I} W_{i} \\
& \sum_{i \in I} s_{i j} \geq \sum_{j \in J} D_{j} / Q \\
& j \in J \\
& l_{i j} \leq Q \cdot s_{i j} \\
& \forall i, j \in V \\
& \sum_{j \in J} l_{i j} \leq W_{i} \cdot y_{i} \quad \forall i \in I \\
& y_{i} \in\{0,1\} \quad \forall i \in I \\
& s_{i j} \in\{0,1\} \quad \forall i, j \in V \\
& l_{i j} \in R \quad \forall i, j \in V
\end{aligned}
$$

The objective function (6) minimizes operating costs, which correspond to the total travelling cost of the radial routes used to deliver the goods to the costumers, the sum of the setup cost of depots and the fixed cost associated with use of each vehicle. The second objective (7) models the fuel consumption and the total emission associated to this fuel consumption. Note that in the optimal solution the routes have a radial configuration in which they start at the depot and end up at a client. Thus, the set of constraints (8) and (9) impose the radial connectivity requirements of the OLRP as described below.

The topology of open routing problems (ORPs) can be considered a graph consisting of $x$ arcs and $y$ nodes $(y=|V|)$. As it is shown in (Lavorato et al., 2012; Bazaraa et al., 2011), in case there is only one depot, it is possible to compare the radial topology of an open problem with a tree, which is a subgraph connected with $x-1$ arcs. For $m$ depots, the radial characteristic requires a subgraph connected with $x-$ $m=|J|$ arcs. Therefore, it can be seen that the radial connectivity requirements impose that the cardinality of $J$ (number of customers) must be equal the number of arcs used in the optimal solution, as is guaranteed by the constraint (8). However, this single condition is not sufficient for radial solutions, since it must ensure proper system connectivity through the set of constraints (9), which guarantees the balance of demand flow in each customer so that it is fully served when visited.

In general, one can state that the topology of an ORP with $y$ nodes is radial if it satisfies the two following conditions:

- Condition 1: the solution must have $x-m$ arcs;

- Condition 2: the solution must be connected. 
Constraint (10) imposes that exactly one arc enters to each vertex associated with a customer. Consequently, the outdegree constraint (11) imposes that exactly one arc leaves each vertex associated with a customer, except for those customers who are at the end of the route. The uni-directional constraint (12) ensures that only one of the two variables $s_{i j}$ or $s_{j i}$ must be used.

Constraints (13) and (14) impose both the depot and vehicle capacity requirements, respectively. Constraints (15) and (16) impose capacity requirements of flow of goods transported. Finally, constraints (17) and (18) define all binary decision variables, and constraint (19) defines the real variable.

\subsection{Multi-objective optimization}

Multi-Objective Optimization (MOO) is the computational process of simultaneously optimizing two or more conflicting objectives subject to a set of constraint functions. In MOO, the main focus is on to produce trade-off solutions representing the best possible compromises among different (possibly conflicting) objectives.

In this paper, we approach the proposed multi-objective problem using an a posteriori methodology, in which the optimization returns some Pareto-optimal solutions, leaving the decision-making process to a post-optimization stage (Marler and Arora, 2009).

In order to solve the multi-objective problem, one can adopt parameterized scalar problems, whose solution leads to a Pareto-optimal solution. For further discussion on multi-objective methods, we refer to (Miettinen, 1999; Ehrgott and Gandibleux, 2002; Marler and Arora, 2009). In the ò-constraint method, one objective is selected to be optimized, while the others are converted into inequality constraints by imposing upper bounds ò.

$$
\begin{aligned}
& \min _{x} \Psi_{1}(x) \\
& \Psi_{k}(x) \leq \varepsilon_{k} \quad \mathrm{k}=2, \ldots, \mathrm{m} \\
& x \in \Omega
\end{aligned}
$$

Cohon and Marks (1975) show that the ò-constraint method can be derived from the Kuhn-Tucker conditions for optimality for a MOO problem. A systematic variation of the parameters $\grave{\mathrm{o}}_{k}$ can yield Pareto optimal solutions (Marler and Arora, 2009). If it exists, a solution to the ò-constraint formulation is weakly Pareto optimal, as shown in (Miettinen, 1999). Moreover, if the solution is unique, then it is Pareto-optimal.

For more than two objectives, the ò-constraint formulation can lead to infeasibility problems, for some combinations of values of $\dot{\mathrm{o}}_{k}$. Nevertheless, for two objectives, as is the case in our formulation, the method can yield Pareto optimal solutions with a systematic variation of $\grave{c}$. In order to generate points on the Pareto front, first we optimize each objective individually with the original constraints of the model and neglecting the other objective. This yields the minimum and maximum values of each objective that contain the Pareto front. Intermediate points on the front are obtained with discrete steps, varying c̀ within the minimum and maximum range. The generation of the Pareto front is independent of which objective is chosen to be minimized. If we select $\Psi_{1}$ as objective and convert $\Psi_{2}$ into a constraint or vice-versa, the result is practically the same, the Pareto front is obtained regardless of the selected objective. Nonetheless, we have observed in our experiments that when the emission $\left(\Psi_{2}\right)$ is used as objective and the operational costs $\left(\Psi_{1}\right)$ are posed as a constraint, the model is solved much faster than when the converse is done, allowing the solution of larger instances.

\section{Computational results}

In this study the test scenarios correspond to instances of the literature to the capacitated location-routing problem presented in (Prins et al., 2007), which include 20, 50, 100 and 200 customers and from 5 to 10 
deposits. The calculation of consumption of the vehicle was taken from the report of University of Michigan: Transportation Research Institute (2014), in which it is established that the average fuel consumption of a vehicle with these characteristics is 1 gallon per $15.81 \mathrm{~km}$ traveled. This value was used as a reference for calculating vehicle consumption at full load, which was estimated at $12 \mathrm{~km}$ per gallon. As for the amount of emissions per gallon of gasoline, we consider $8.70645 \mathrm{~kg}$ of $\mathrm{CO}_{2}$ per gallon, this information is related to the fuel consumption guide (Gouvermment of Canada, 2015). In this report $\mathrm{CO}_{2}$ emissions vary according to the type of fuel used and engine characteristics such as size, type, vehicle brand and optimum cruising speed. The cost of emissions is calculated based on quoted prices presented in (SENDECO2, 2014). The value of 0.009 USD per $\mathrm{kg}$ of $\mathrm{CO}_{2}$. To quantify the price of a gallon of gasoline was consulted online ${ }^{1}$. This information is set according to the territory where the case study is contemplated. In our case, this parameter was 3.92 USD per gallon. The mathematical model was solved under CPLEX 12.5 (ILOG, 2008) on a computer Intel Core i7-4770 3.4 GHz, 16 GB of RAM and written in AMPL: A Modeling Language for Mathematical Programming (Fourer et al., 2002). The method used to generate the Pareto front is the ò-constraint method, using $\Psi_{2}$ as objective and $\Psi_{1}$ as a constraint. Sixteen instances used by Prins et al. (2007) are used in order to verify the efficacy of the proposed model and to confirm the observation that by increasing the number of vehicles the fuel consumption and hence total emission can be reduced, in the context of OLRP considering fuel consumption minimization.

In all the instances analyzed, we have obtained a Pareto front representing the trade-off between greenhouse gases emissions and operational costs. In order to better analyze the characteristics of the solutions on the Pareto front and to get better insights about the nature of this trade-off, we compare in Fig. 1 three representative points from the Pareto front obtained for the instance $100102 \mathrm{~b}$. Point A corresponds to the minimization of $\Psi_{1}$ (operational costs), point $\mathrm{C}$ corresponds to the minimization of $\Psi_{2}$ (environmental impact) and point B corresponds to the solution selected by the min-max criterion (or minimization of maximum regret), which usually corresponds to a middle point on the Pareto front, a point that minimizes the maximum deviation to the minimum of each objective. Interesting characteristics can be observed from the analysis of Fig. 1. Point $\mathrm{A}$ is a solution characterized by minimum number of depots and vehicles, leading to longer routes connecting the clients to the depots. This solution involves employing less vehicles, operating fully loaded under longer routes, which in turn generates greater fuel consumption and consequently greater emission of green-house gases. With less vehicles, the amount of cargo per vehicle increases, which affects directly the fuel consumption, leading to a high value in the objective function $\Psi_{2}$.

Point C, on the other extreme of the Pareto front, is related to the minimization of objective function $\Psi_{2}$ (environmental impact). This solution presents a greater number of depots and routes than the solution of point A. In this way, the routes that connect the customers to the depots are shorter on average, implying shorter paths and fast deliveries of the load. As a consequence, the vehicles presents less fuel consumption overall and less emission of green-house gases. It is intuitive that more fuel will be consumed if more vehicles are used. However, in the context of CLRP considering fuel consumption minimization, generally using more vehicles does not imply lengthening the travel distance. It is interesting to notice that by increasing the number of vehicles the fuel consumption and hence total emission can be reduced. Using few vehicles at full capacity does not necessarily imply in fuel economy, whereas using more vehicles, not fully loaded, can translate into reduced fuel cost and less environmental impact.

In the long term, by having more depots and more routes, the fuel economy can balance out the initial investment costs. Another aspect influencing the minimization of emission is that the vehicles tend to be dispatched to attend those clients with higher demand. Heavier loads are delivered earlier in the route, decreasing the weight of the vehicle and contributing to the reduction of emissions. 


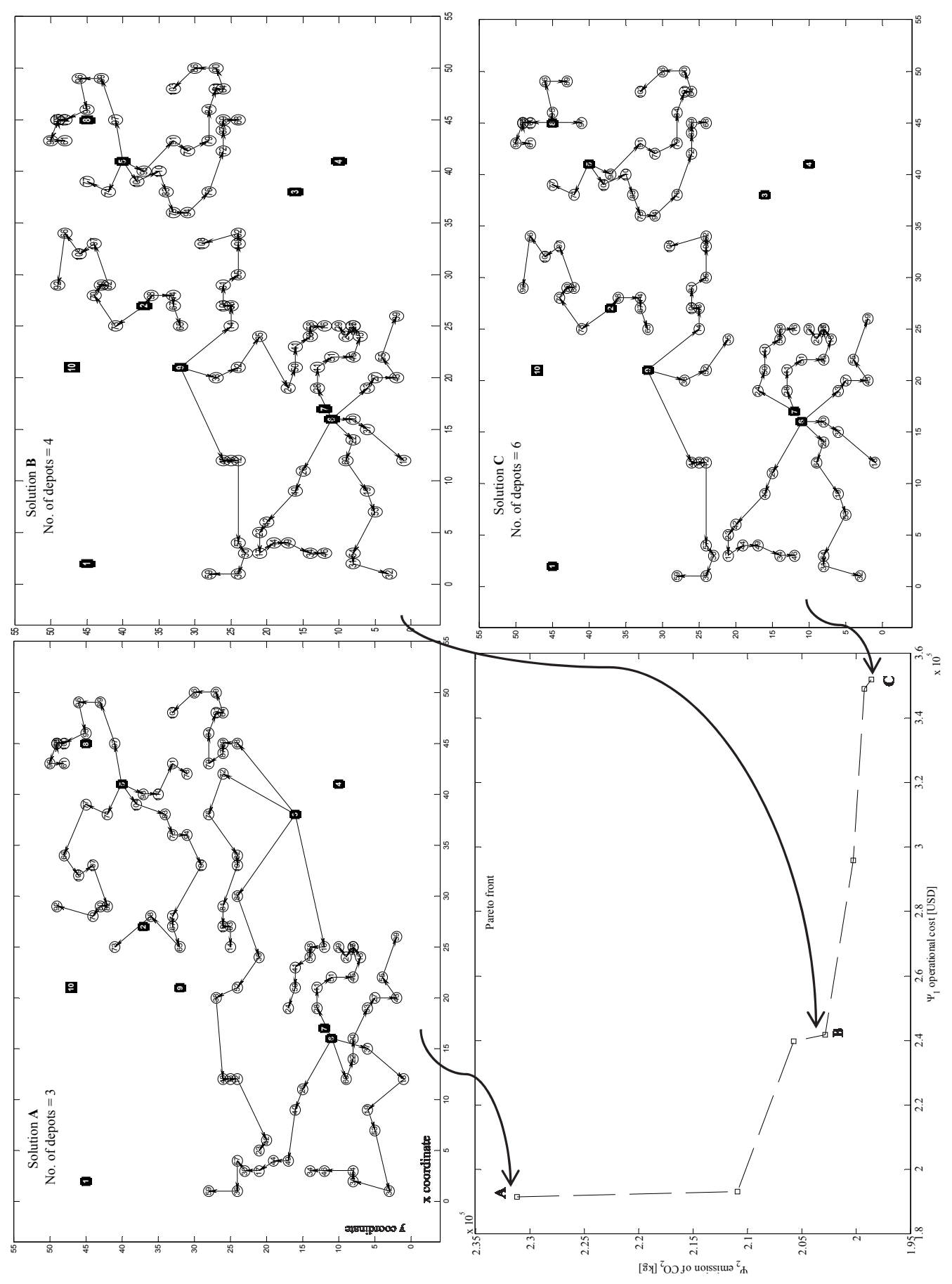

Fig. 1. Pareto front and three representative optimal solutions for the instance 100_10_2b used by Prins et al. (2007)

Point B was selected according to the min-max criterion. This solution is characterized by an medium number of depots and vehicles with medium length paths. Similarly to the previous cases, it is possible to note how the number of depots affects the length of the paths connecting customers and depots. This solution corresponds to a balance between operational costs and total emissions. The reduction of operational costs favors solutions with longer routes, few vehicles and depots. From the perspective of environmental impact, the reduction of emissions leads to solutions with more vehicles and shorter 
routes, usually attending clients with higher demand first. Other solutions along the Pareto front represent different trade-off configurations between these two objectives.

\section{Table 1}

Computational results for instances used by Prins et al. (2007)

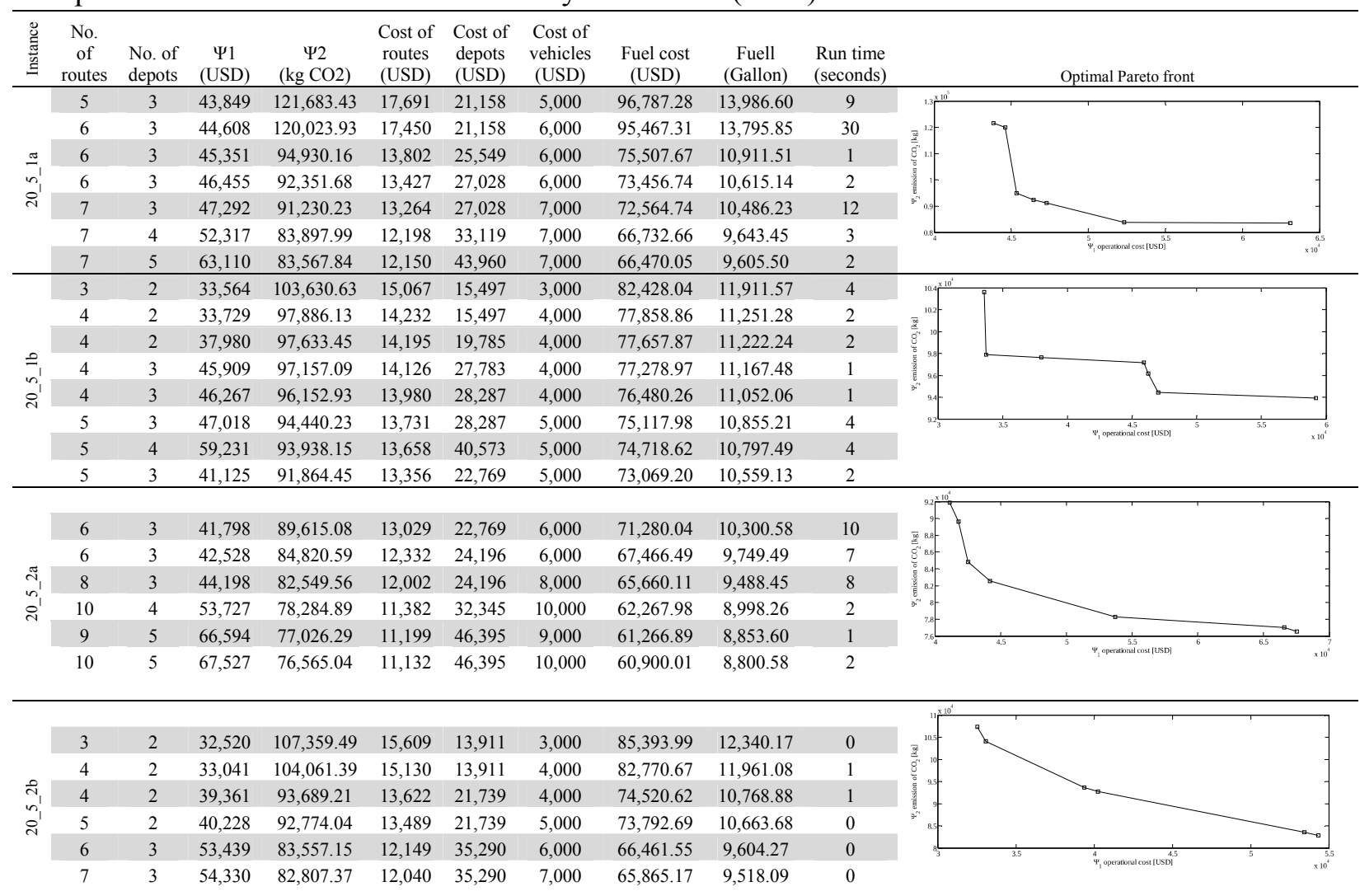

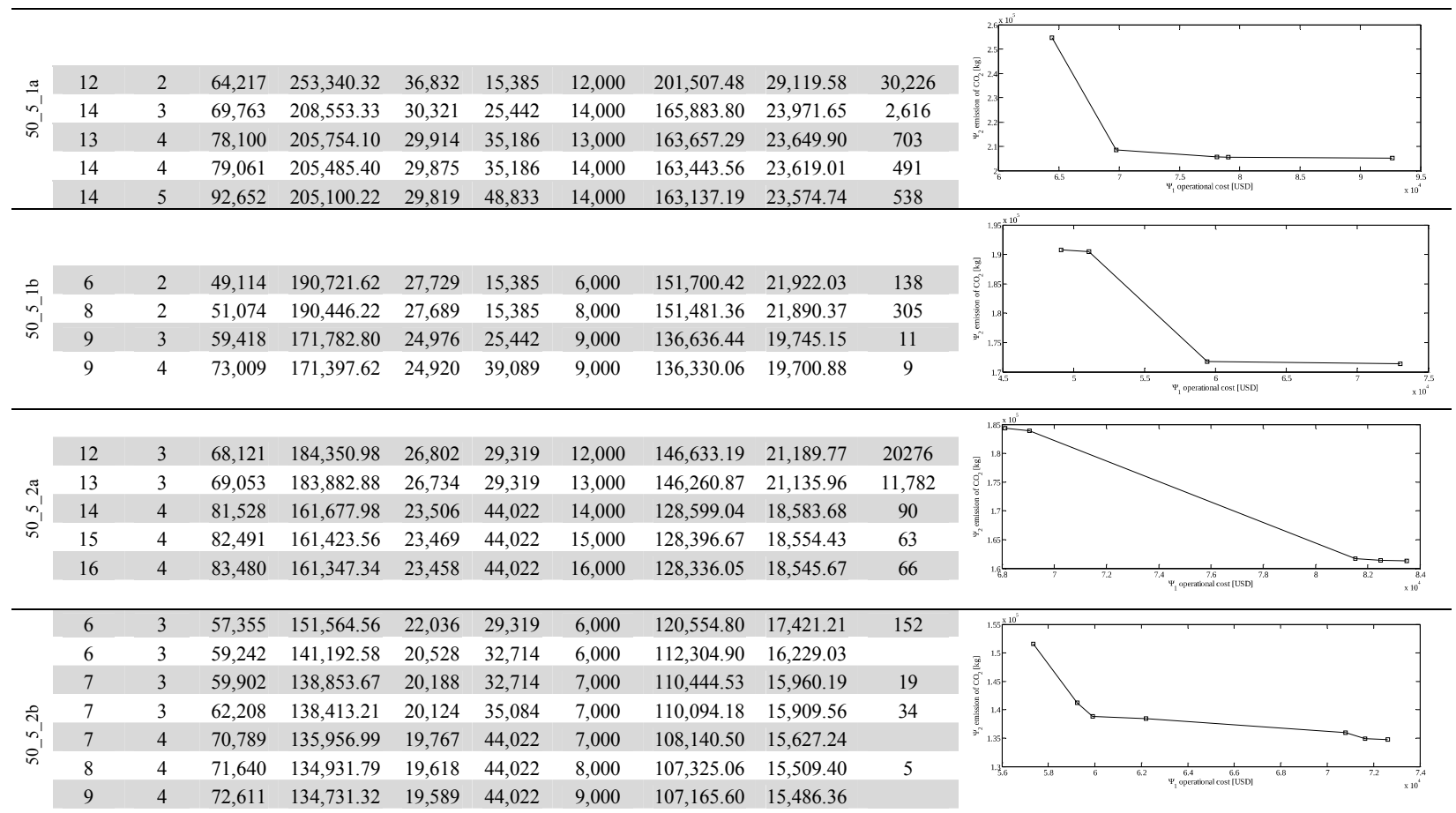


Table 1

Computational results for instances used by Prins et al. (2007) (Continued)

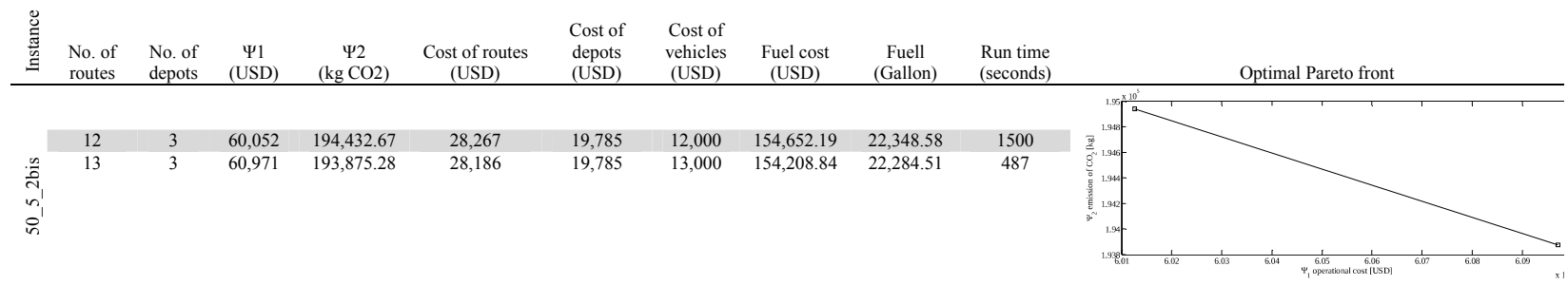

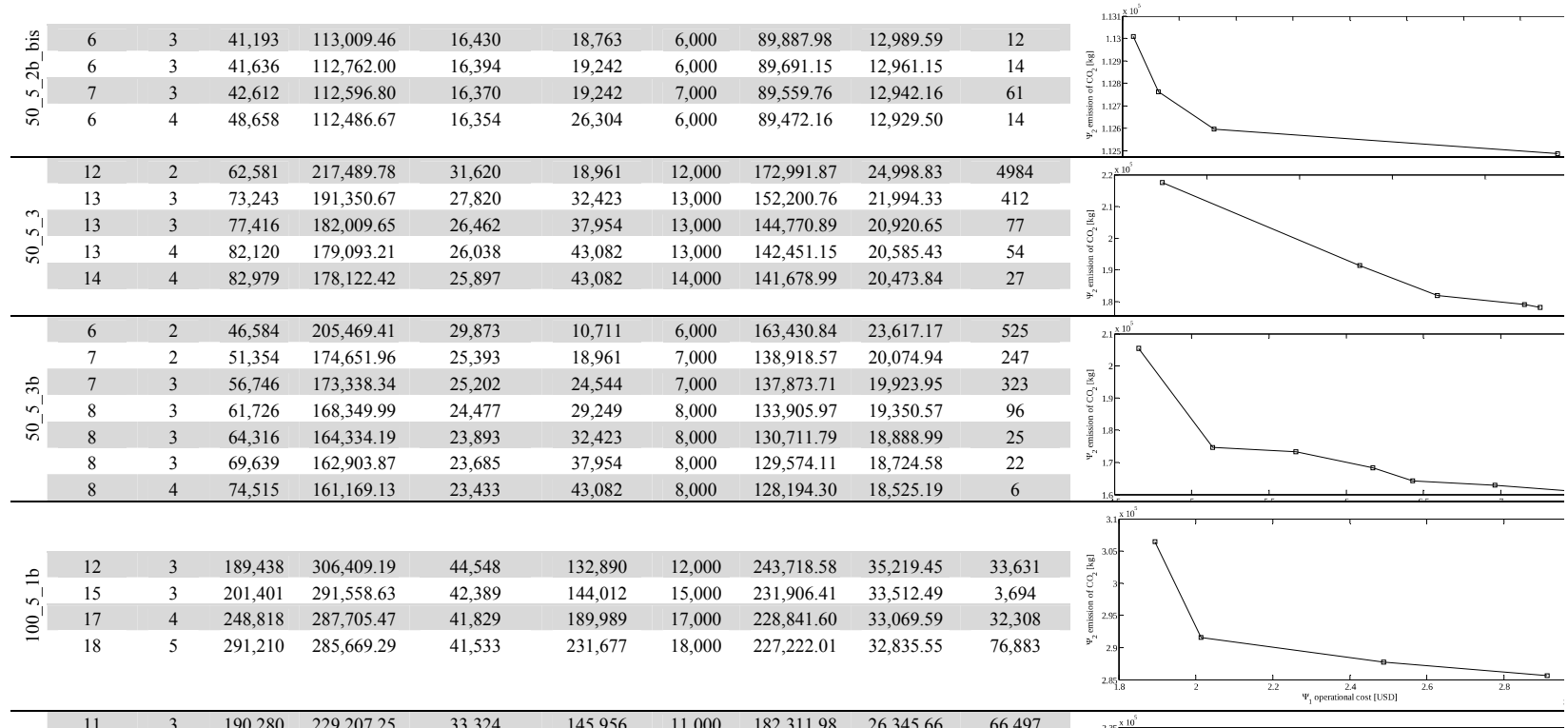

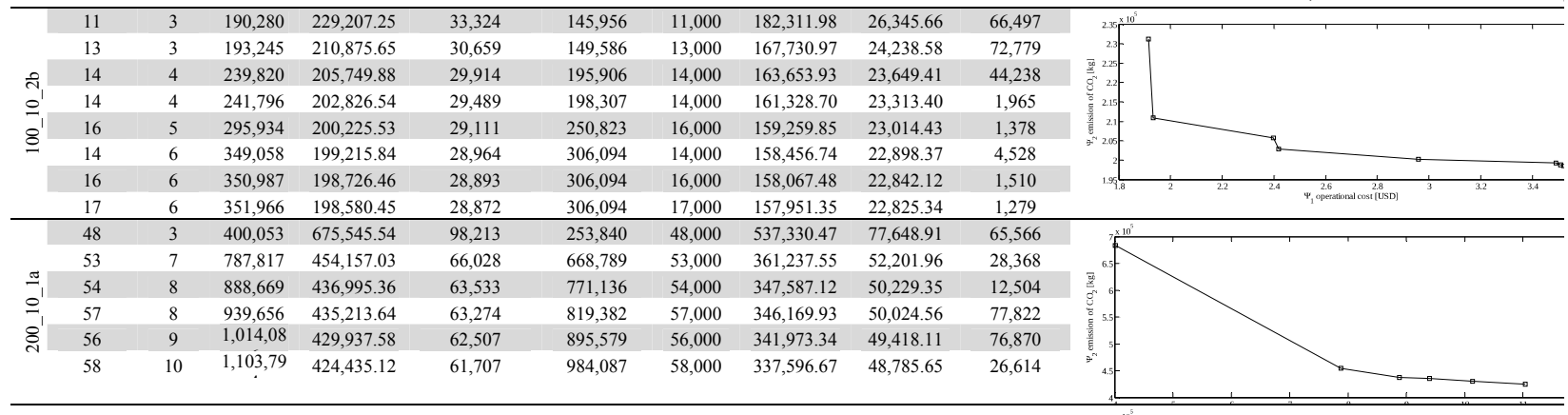

\begin{tabular}{|c|c|c|c|c|c|c|c|c|c|}
\hline 23 & 3 & 350,257 & $322,497.10$ & 46,887 & 280,370 & 23,000 & $256,514.93$ & $37,068.63$ & 71,118 \\
\hline 27 & 6 & 692,466 & $280,542.94$ & 40,788 & 624,678 & 27,000 & $223,144.50$ & $32,246.31$ & 53,679 \\
\hline 27 & 7 & 816,443 & $277,199.73$ & 40,302 & 749,141 & 27,000 & $220,485.30$ & $31,862.04$ & 30,562 \\
\hline 27 & 7 & 816,389 & $276,828.46$ & 40,248 & 749,141 & 27,000 & $220,189.99$ & $31,819.36$ & 33,143 \\
\hline
\end{tabular}

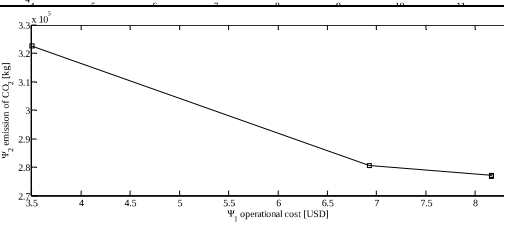

Table 1 presents the results obtained with the proposed model in 16 instances. Columns 2-5 show information about the optimal solution in three different points on the Pareto front: number of routes, number of depots, value of objective function $\Psi_{1}$, value of objective function $\Psi_{2}$. Columns 6-8 present the discrimination of the three terms in the first objective of operational costs, which correspond to the total travelling cost of the radial routes used to deliver the goods to the costumers, the sum of the setup cost of depots and the fixed cost associated with the use of each vehicle, respectively. Columns 9-10 present the discrimination of the terms in the second objective of emission $\Psi_{2}$, which correspond to the fuel consumption and the total emission associated to this fuel consumption. Column 11 reports the required computational time and column 12 shows the points on the Pareto front obtained with the $q$ constraint method. This last column also allows us to conclude that the proposed model is able to generate a set of trade-off solutions for the problem. In the results, we were able to obtain solutions with GAP 
equal to zero for instances up to 20 customers. In instances with 50 customers, most solutions obtained have GAP equal to zero and few solutions were obtained with GAP less than 3\%. In instances with 100 customers, solutions were obtained with GAP inferior to $2 \%$ and in instances with 200 customers, with GAP less than 3\%. We have observed in our experiments that when the emission $\left(\Psi_{2}\right)$ is used as objective and the operational costs $\left(\Psi_{1}\right)$ are posed as a constraint, the model is solved much faster than when the converse is done, allowing the solution of larger instances. Usually, when minimizing emissions, the number of selected depots is greater and therefore the complexity of the problem is lower, what reflects on the computation time required for the solution. Finally, it is interesting to highlight that the solutions obtained with the relaxation of $\left(\Psi_{1}\right)$ correspond to the Pollution-Routing Problem (PRP). Therefore, the results reported here can be considered as a reference for comparison with the single objective PRP.

\section{Conclusions and future work}

This paper proposed a multi-objective model for the G-OLRP considering fuel consumption. The model proposed is a bi-objective problem, considering the minimization of operational costs and the minimization of environmental effects. The proposed model for the calculation of particle emission was developed under assumptions close to the real scenario, assuming constant speed. The model can be adjusted for more complicated scenarios, for instance, considering an average inclination between any pair of nodes, varying speed, conditions of the road, heterogeneous fleet, among others. These variations will affect the values of the parameters $\alpha$ and $\gamma$, possibly with different values for each arc.

The use of radiality constraints in the solution of open VRP has been very effective, which was motivated by the radial topology of open routes. The proposed mathematical model can serve as a reference to the solution of larger instances, in which other strategies can be employed, such as hybrid methods, heuristics, meta-heuristics, math-heuristics, set partitioning. The multi-objective approach to this problem is entirely appropriate, since the trade-off between economic aspects and environmental aspects can be selected from the Pareto front using a decision-making process. The fuel consumption is affected by the number of depots and the number of vehicles. The use of more vehicles implies a better distribution of the cargo per vehicle. However, when reducing emissions, one can find short routes, vehicles traveling with moderate load and delivering higher loads first. From the point of view of environmental impact, more vehicles performing shorter routes and serving as soon as possible those customers with higher demand seems to be the preferred strategy. In future work we should explore other methodologies for the calculation of fuel consumption and should consider aspects such as the slope of the road and vehicle speed in different edges.

\section{Acknowledgements}

E.M. Toro, M. Granada and R.A. Gallego would like to thank the support given by the Technological University of Pereira, Colombia.

F. G. Guimarães would like to thank the support given by the National Council for Scientific and Technological Development (CNPq grant no. 312276/2013-3) and Fundação de Amparo à Pesquisa do Estado de Minas Gerais (FAPEMIG), Brazil.

\section{References}

Barreto, S., Ferreira, C., Paixao, J., \& Santos, B. S. (2007). Using clustering analysis in a capacitated location-routing problem. European Journal of Operational Research, 179(3), 968-977.

Bazaraa, M. S., Jarvis, J. J., \& Sherali, H. D. (2011). Linear programming and network flows. John Wiley \& Sons.

Bektaş, T., \& Laporte, G. (2011). The pollution-routing problem. Transportation Research Part B: Methodological, 45(8), 1232-1250.

Bodin, L., Golden, B., and Assad, A. (1983). Routing and scheduling of vehicles and crews: the state of the art. Computers and Operations Research, 10(2), $63-211$. 
Boriboonsomsin, K., Vu, A., \& Barth, M. (2010). Eco-driving: pilot evaluation of driving behavior changes among us drivers. University of California Transportation Center.

Braekers, K., Ramaekers, K., \& Van Nieuwenhuyse, I. (2015). The vehicle routing problem: State of the art classification and review. Computers \& Industrial Engineering, 99, 300-313.

Cohon, J. L., \& Marks, D. H. (1975). A review and evaluation of multiobjective programing techniques. Water Resources Research, 11(2), 208-220.

Daniel, S. E., Diakoulaki, D. C., \& Pappis, C. P. (1997). Operations research and environmental planning. European Journal of Operational Research, 102(2), 248-263.

Demir, E., Bektaş, T., \& Laporte, G. (2012). An adaptive large neighborhood search heuristic for the pollution-routing problem. European Journal of Operational Research, 223(2), 346-359.

Demir, E., Bektaş, T., \& Laporte, G. (2014). The bi-objective pollution-routing problem. European Journal of Operational Research, 232(3), 464-478.

Dueck, G., \& Scheuer, T. (1990). Threshold accepting: a general purpose optimization algorithm appearing superior to simulated annealing. Journal of Computational Physics, 90(1), 161-175.

Ehrgott, M., \& Gandibleux, X. (2003). Multiobjective combinatorial optimization-theory, methodology, and applications. In Multiple criteria optimization: State of the art annotated bibliographic surveys (pp. 369-444). Springer US.

Erdogan, S. and Miller-Hooks, E. (2012). A green vehicle routing problem. Transportation Research Part E: Logistics and Transportation Review, 48(1), 100-114.

Figliozzi, M. A. (2010). An iterative route construction and improvement algorithm for the vehicle routing problem with soft time windows. Transportation Research Part C: Emerging Technologies, 18(5), 668-679.

Fourer, R., Gay, D. M., \& Kernighan, B. W. (2002). AMPL: A Modeling Language for Mathematical Programming. Brooks/Cole-Thomson, 2nd edition.

Neto, J. Q. F., Walther, G., Bloemhof, J., Van Nunen, J. A. E. E., \& Spengler, T. (2009). A methodology for assessing eco-efficiency in logistics networks.European Journal of Operational Research, 193(3), 670-682.

Golden, B. L., Raghavan, S., \& Wasil, E. A. (Eds.). (2008). The vehicle routing problem: latest advances and new challenges (Vol. 43). Springer Science \& Business Media.

Gouvermment of Canada (2015). Fuel consumption guide.

Ho, W., Ho, G. T., Ji, P., \& Lau, H. C. (2008). A hybrid genetic algorithm for the multi-depot vehicle routing problem. Engineering Applications of Artificial Intelligence, 21(4), 548-557.

ILOG, S. (2008). CPLEX optimization subroutine library guide and reference.System v11. 0 User's Guide.

Jemai, J., Zekri, M., \& Mellouli, K. (2012, April). An NSGA-II algorithm for the green vehicle routing problem. In European Conference on Evolutionary Computation in Combinatorial Optimization (pp. 37-48). Springer Berlin Heidelberg.

Kara, I., Kara, B. Y., \& Yetis, M. K. (2007, August). Energy minimizing vehicle routing problem. In International Conference on Combinatorial Optimization and Applications (pp. 62-71). Springer Berlin Heidelberg.

Kucukoglu, I., Ene, S., Aksoy, A., \& Ozturk, N. (2013). Green capacitated vehicle routing problem fuel consumption optimization model. Computational Engineering Research, 3, 16-23.

Lalla-Ruiz, E., Expósito-Izquierdo, C., Taheripour, S., \& Voß, S. (2016). An improved formulation for the multi-depot open vehicle routing problem. OR Spectrum, 38(1), 175-187.

Lavorato, M., Franco, J. F., Rider, M. J., \& Romero, R. (2012). Imposing radiality constraints in distribution system optimization problems. IEEE Transactions on Power Systems, 27(1), 172-180.

Lin, C., Choy, K. L., Ho, G. T., Chung, S. H., \& Lam, H. Y. (2014). Survey of green vehicle routing problem: past and future trends. Expert Systems with Applications, 41(4), 1118-1138.

Liu, R., \& Jiang, Z. (2012). The close-open mixed vehicle routing problem.European Journal of Operational Research, 220(2), 349-360.

Marler, R. T., \& Arora, J. S. (2009). Multi-objective optimization: concepts and methods for engineering. VDM Publishing. 
Miettinen, K. (1999). Nonlinear Multiobjective Optimization, volume 12 of International Series in Operations Research and Management Science.

Mirabi, M., Ghomi, S. F., \& Jolai, F. (2010). Efficient stochastic hybrid heuristics for the multi-depot vehicle routing problem. Robotics and Computer-Integrated Manufacturing, 26(6), 564-569.

Palmer, A. (2007). The Development of an Integrated Routing and Carbon Dioxide Emissions Model for Goods Vehicles. PhD thesis, School of Management, Cranfield University.

Pradenas, L., Oportus, B., \& Parada, V. (2013). Mitigation of greenhouse gas emissions in vehicle routing problems with backhauling. Expert Systems with Applications, 40(8), 2985-2991.

Prins, C., Prodhon, C., \& Calvo, R. W. (2006). Solving the capacitated location-routing problem by a GRASP complemented by a learning process and a path relinking. 4OR, 4(3), 221-238.

Prins, C., Prodhon, C., Ruiz, A., Soriano, P., \& Wolfler Calvo, R. (2007). Solving the capacitated location-routing problem by a cooperative Lagrangean relaxation-granular tabu search heuristic. Transportation Science, 41(4), 470-483.

Schrage, L. (1981). Formulation and structure of more complex/realistic routing and scheduling problems. Networks, 11(2), 229-232.

SENDECO2 (2014). The European bourse for European Unit Allowances ( EUA ) and Carbon Credits ( CER's ).

Suzuki, Y. (2011). A new truck-routing approach for reducing fuel consumption and pollutants emission. Transportation Research Part D: Transport and Environment, 16(1), 73-77.

Tarantilis, C. D., \& Kiranoudis, C. T. (2002). Distribution of fresh meat. Journal of Food Engineering, 51(1), 85-91.

Toro, E., Escobar, A., \& Granada, M. (2016). Literature Review on the Vehicle Routing Problem in the Green Transportation Context. Luna Azul, 42(1), 362 - 387.

Toth, P., \& Vigo, D. (Eds.). (2014). Vehicle routing: problems, methods, and applications (Vol. 18). Siam.

Transportation Research Institute (2014). Large drop in fuel economy in september.

Trigeorgis, L. (1996). Real options: Managerial flexibility and strategy in resource allocation. MIT press.

Tuzun, D., \& Burke, L. I. (1999). A two-phase tabu search approach to the location routing problem. European journal of operational research, 116(1), 87-99.

Ubeda, S., Arcelus, F. J., \& Faulin, J. (2011). Green logistics at Eroski: A case study. International Journal of Production Economics, 131(1), 44-51.

Xiao, Y., Zhao, Q., Kaku, I., \& Xu, Y. (2012). Development of a fuel consumption optimization model for the capacitated vehicle routing problem.Computers \& Operations Research, 39(7), 1419-1431.

Yao, B., Hu, P., Zhang, M., \& Tian, X. (2014). Improved ant colony optimization for seafood product delivery routing problem. PROMET-Traffic\&Transportation,26(1), 1-10.

Vincent, F. Y., \& Lin, S. Y. (2015). A simulated annealing heuristic for the open location-routing problem. Computers \& Operations Research, 62, 184-196.

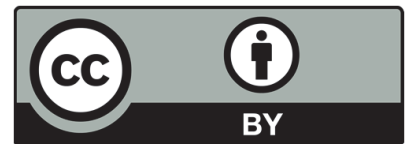

(C) 2016 by the authors; licensee Growing Science, Canada. This is an open access article distributed under the terms and conditions of the Creative Commons Attribution (CCBY) license (http://creativecommons.org/licenses/by/4.0/). 